\title{
EFEITOS DA APLICACCÃO DE HERBICIDAS SOBRE A EFICIÊNCIA FOTOQUÍMICA EM PLANTAS DE SOJA CONVENCIONAL E GENETICAMENTE MODIFICADA
}

\author{
Effects of herbicides application on photochemical efficiency in \\ conventional and genetically modified soybeans
}

\author{
Maria José Pinheiro Corrêa ${ }^{1}$ Pedro Luís da Costa Aguiar Alves ${ }^{2}$
}

\begin{abstract}
RESUMO
Objetivou-se, neste trabalho, avaliar o efeito da aplicação de herbicidas em pós-emergência sobre a eficiência fotoquímica de duas cultivares de soja (M-SOY 7908 RR e M-SOY 8001). O delineamento experimental utilizado foi em blocos ao acaso, com quatro repetições. Os tratamentos constaram da aplicação de herbicidas isolados e em misturas: lactofen, glyphosate, lactofen + chlorimuron-ethyl, chlorimuron-ethyl + imazethapyr, chlorimuron-ethyl + bentazon, glyphosate + imazethapyr, lactofen + chlorimuron-ethyl + imazethapyr e lactofen + chlorimuron-ethyl + imazethapyr/haloxyfop-methyl. Além disso, foram mantidas duas testemunhas sem aplicação de herbicida. As avaliações foram realizadas aos 4; 11; 18; 25 e 32 dias após aplicação dos herbicidas (DAA) no primeiro ano, e aos 6, 14, 21, 28 e 35 DAA, no segundo. A cultivar M-SOY 8001 foi suscetível à aplicação dos herbicidas, principalmente às misturas contendo lactofen + chlorimuron-ethyl e lactofen + chlorimuron-ethyl + imazethapyr + haloxifop-methyl, os quais provocaram redução no rendimento quântico máximo do PSII (Fv/Fm). As duas cultivares apresentaram suscetibilidade aos herbicidas quando tratados com lactofen aplicado isolado e nas misturas lactofen + chlorimuron-ethyl, chlorimuronethyl + imazethapyr, lactofen + chlorimuron-ethyl + imazethapyr e lactofen + chlorimuron-ethyl + imazethapyr + haloxifopmethyl com redução nos teores de clorofila, estimados pelo Medidor Portátil de Clorofilas. Os danos foram reversíveis, desaparecendo gradativamente com a idade das plantas.
\end{abstract}

Termos para indexação: Clorofila, fluorescência, clorofilômetro.

\section{ABSTRACT}

The objective of this work was to evaluate the influence of post-emergency herbicide application on the photosynthetic activity of two soybean cultivars (M-SOY 7908 RR and M-SOY 8001). A randomized block experimental design was used with four replicates. Treatments consisted of applied herbicide mixtures or herbicides only: lactofen, glyphosate, lactofen + chlorimuron-ethyl, chlorimuron-ethyl + imazethapyr, chlorimuron-ethyl + bentazon, glyphosate + imazethapyr, lactofen + chlorimuron-ethyl + imazethapyr, lactofen + chlorimuron-ethyl + imazethapyr / haloxyfop-methyl. The tests included two control. The evaluations were taken after the application of herbicides at 4;11;18;25 and 32 in the first crop and at $6,14,21,28$ and $35 \mathrm{DAA}$ in the second crop. The M-SOY 8001 was susceptible the herbicides application mostly the herbicide mixture lactofen + chlorimuron-ethyl and lactofen + chlorimuron-ethyl + imazethapyr + haloxifop-methyl the herbicides caused reduction in maximum quantum efficiency of PSII $(\mathrm{Fv} / \mathrm{Fm})$. However, both varieties were susceptible to the treatments lactofen alone applied and the mixtures lactofen + chlorimuron-ethyl, chlorimuron-ethyl + imazethapyr, lactofen + chlorimuron-ethyl + imazethapyr and lactofen + chlorimuron-ethyl + imazethapyr + haloxifop-methyl with reduction in chlorophyll relative content by portable chlorophyll meter. However, these damages were reversible, disappearing gradually with plant growth.

Index terms: Chlorophyll, fluorescence, chlorophyll meter.

(Recebido em 18 de junho de 2009 e aprovado em 25 de fevereiro de 2010)

\section{INTRODUÇÃO}

A soja, assim como outra cultura de interesse agronômico, apresenta problemas relacionados à competição das plantas daninhas, e atualmente, a forma mais empregada para minimizar esse problema é o emprego de herbicidas. Os herbicidas controlam o crescimento e o desenvolvimento das plantas daninhas, por meio da ação desses compostos em processos metabólicos e fisiológicos vitais dessas classes de plantas. Geralmente, os herbicidas inibem a atividade de uma enzima/proteína na célula e, como consequência, desencadeiam uma série de eventos que podem matar ou inibir o metabolismo celular, e consequentemente do organismo (Melhorança \& Pereira, 2000). Segundo o Herbicide Resistance Action

\footnotetext{
${ }^{1}$ Universidade Estadual do Maranhão/UEMA - Departamento de Química e Biologia - Campus Universitário Paulo VI - s/n. Tirirical - Cx. P. 09 65055-310 - São Luís, MA - mjcorreazea@hotmail.com

2Universidade Estadual Paulista/UNESP - Departamento de Biologia Aplicada à Agropecuária - Jaboticabal,SP
} 
Committee - HRAC (2008), os herbicidas são classificados alfabeticamente, de acordo com seus sítos de ação, modo de ação, similaridade de sintomas ou classes químicas. No caso de herbicidas inibidores de fotossíntese, esses são classificados em C1, C2 e C3 (inibição da fotossíntese, via ação no Fotossistema II) e D (inibição da fotossíntese, via ação no Fotossistema I). Os herbicidas do grupo C possuem o mesmo modo de ação, mas possuem respostas distintas quanto à ligação à proteína D1.

A fotossíntese é o processo de conversão da energia luminosa em energia química, e é responsável pela incorporação de biomassa das plantas. Esse porcesso é extremamente dependente dos fatores do ambiente como luz, temperatura, $\mathrm{CO} 2$, água e disponibidade de nutrientes minerais. Durante a fotossíntese, parte da energia luminosa absorvida pelos pigmentos cloroplastídicos da folha é reemitida como fluorescência (Glynn et al., 2003). As medidas da emissão da fluorescência da clorofila, principalmente em relação ao fotossistema II (PSII), e a estimativa do teor relativo de clorofila por meio do medidor portátil de clorofilas têm sido utilizadas para obter informações qualitativas e quantitativas rápidas sobre o processo fotossintético, com a vantagem de serem determinações não destrutivas, precisas e rápidas.

A razão de fluorescência $\mathrm{Fv} / \mathrm{Fm}$, que indica a eficiência quântica máxima do Fotossistema II (PSII), é usada como um indicador da capacidade fotossintética nas plantas, e tem se tornado uma importante característica fisiológica nos estudos relacionados aos vários tipos de estresses (Krause \& Weiss, 1991). Se a planta estiver em condições normais, não estressantes, na maioria das espécies, o valor dessa variável pode variar de 0,75 a 0,85 . Os valores inferiores a esses indicarão comprometimentos na eficiência quântica máxima do fotossistema II e, por consequência, do potencial fotossintético da planta (Maxwell \& Johnson, 2000). Quando as plantas são submetidas a estresses como seca (Ogren \& Oquist, 1985), aplicação de herbicidas (Catunda et al., 2005) e salinidade (Zanandrea et al., 2006), o declínio em Fv/Fm é um bom indicador de dano no aparato fotoquímico das plantas.

A determinação do teor relativo de clorofila total por meio de um medidor portátil permite medições instantâneas de valor correspondente ao seu teor na folha sem destruí-la e tem sido utilizado para estimar o conteúdo de clorofila em um grande número de espécies de plantas (Argenta et al., 2001; Zotarelli et al., 2003; Benett et al., 2008; Rozane et al., 2009). O medidor portátil de clorofila, SPAD (Soil Plant Analysis Development), mede a transmissão de luz vermelha a $650 \mathrm{~nm}$ e de luz infra vermelha a $940 \mathrm{~nm}$, sem absorção. Com base nesses valores, o equipamento calcula o valor ou índice SPAD, o qual é correlacionado positivamente com o teor de clorofila (Silveira et al., 2003). O teor de clorofila total está diretamente correlacionado às propriedades de fluorescência das moléculas de clorofila (Krause \& Weiss, 1991).

Catunda et al. (2005) constataram que o herbicida amicarbazone promoveu o declínio nos teores de pigmentos fotossintéticos em folhas de abacaxi. Entretanto, houve certa seletividade desse herbicida à cultura. No entanto, diuron + paraquat foi letal para a cultura, haja vista a redução nos teores de clorofila $a$ e $b$ e carotenóides. Dessa forma, os autores concluíram que o tratamento diuron + paraquat causou maiores danos ao aparelho fotossintético das plantas.

Realizou-se, este trabalho, com o objetivo de avaliar o efeito da aplicação de herbicidas em pós-emergência sobre a eficiência fotoquímica em plantas de soja convencional (M-SOY 8001) e transgênica (M-SOY 7908 RR).

\section{MATERIAL E MÉTODOS}

O experimento foi realizado em condição de campo, nos anos agrícolas 2006/2007 e 2007/2008, em duas áreas experimentais da Fazenda de Ensino, Pesquisa e Produção. Esse local pertence à Faculdade de Ciências Agrárias e Veterinárias da Universidade Estadual Paulista - UNESP, localizada no município de Jaboticabal - SP (2115'22' S, $48^{\circ} 18^{\prime} 58^{\prime}$ 'W) e altitude de $595 \mathrm{~m}$.

O solo da área experimental do primeiro ano agrícola é classificado como Latossolo Vermelho eutroférrico típico, textura muito argilosa A moderado e relevo suave ondulado. O do segundo é classificado como Latossolo Vermelho eutrófico típico, textura argilosa A moderado, relevo suave ondulado (Andriolli \& Centurion, 1999).

A adubação consistiu da aplicação de $300 \mathrm{~kg} \mathrm{ha}^{-1}$ de $0-20-20$ e $8-20-20$ no sulco de semeadura no primeiro e segundo ano, respectivamente. A soja trangênica (M-SOY 7908 RR) e convencional (M-SOY 8001) foram semeadas em 8 de dezembro de 2006 e 15 de dezembro de 2007, para o primeiro e segundo ano, respectivamente. A semeadura foi realizada com semeadora-adubadora, distribuindo-se 18 e 21 sementes por metro no primeiro e segundo ano, respectivamente. Antes da semeadura e nas duas áreas, procedeu-se ao tratamento das sementes com Thiram (300 ml $100 \mathrm{~kg}^{-1}$ de sementes) e inoculação com $2 \mathrm{~g}$ de inoculante turfoso por $\mathrm{kg}$ de semente.

Os tratamentos constaram da aplicação de herbicidas isolados e em misturas (Tabela 1) e as testemunhas sem aplicação de herbicida (testemunha capinada e mantida infestada). A utilização de mistura de herbicidas deve-se ao fato dessa prática estar se tornando cada vez frequente, uma vez que essas misturas têm 
demonstrado um aumento do espectro de controle de plantas daninhas e, em alguns casos, uma maior eficiência, devido ao efeito sinérgico dos herbicidas.

O delineamento experimental foi em blocos casualizados com dez tratamentos para cada cultivar, e quatro repetições. As parcelas experimentais foram constituídas por sete linhas de semeadura, espaçadas de $0,45 \mathrm{~m}$, com $5 \mathrm{~m}$ de comprimento cada, resultando numa área de $15,75 \mathrm{~m}^{2}$.

Os herbicidas foram aplicados quando a soja se encontrava com o quinto ou sexto (estádio V6 e V7) e quarto ou quinto (estádio V5 e V6) trifólio totalmente expandido, segundo escala fenológica proposta por Ritchie et al. (1977), no primeiro e segundo ano, respectivamente. O herbicida haloxifop-methyl foi aplicado em seguida da pulverização da mistura lactofen + chlorimuron-ethyl + imazethapyr para que sua absorção não fosse prejudicada. Foi utilizado pulverizador costal, à pressão constante (mantida pelo $\mathrm{CO}_{2}$ comprimido) de $2,24 \mathrm{kgf} \mathrm{cm}^{-2}$, munido de barra com seis pontas de pulverização tipo jato plano "Teejet" XR 110.02, espaçados de 0,5 m, com consumo de calda equivalente a $200 \mathrm{~L} \mathrm{ha}^{-1}$. No momento da aplicação, registrou-se para o primeiro e segundo ano agrícola, respectivamente, $34^{\circ} \mathrm{C}$ e $33^{\circ} \mathrm{C}$ de temperatura do ar; $28^{\circ} \mathrm{C}$ e $31^{\circ} \mathrm{C}$ de temperatura do solo; $72 \%$ e $52 \%$ de umidade relativa do ar; ventos leves com velocidade de $2,0 \mathrm{~km} \mathrm{~h}^{-1} \mathrm{e}$ nebulosidade em torno de $80 \%$ e $30 \%$.

Aos $4 ; 11 ; 18 ; 25$ e 32 dias após aplicação (DAA) dos herbicidas, e aos 6, 14, 21 e 28 DAA, no primeiro e segundo ano, respectivamente, foram avaliados a emissão da fluorescência da clorofila (Fv/Fm) e o teor relativo de clorofila. As medições de Fv/Fm foram realizadas por meio da utilização de um fluorômetro portátil (PEA - Hansatech,) em que a folha foi pré-adaptada ao escuro, com a utilização de pinças, por 15 minutos antes das determinações de fluorescência. $\mathrm{O}$ teor relativo de clorofila foi estimado por meio do medidor portátil de clorofilas (MPC) (SPAD 502, Minolta). Essas determinações foram realizadas no folíolo central do trifólio completamente expandido e sempre se utilizou a mesma planta em cada avaliação, fazendo-se uma leitura por folha.

Os dados obtidos foram submetidos à análise de variância pelo teste $\mathrm{F}$ e as médias foram comparadas pelo teste de Tukey, a 5\% de probabilidade.

\section{RESULTADOS E DISCUSSÃO}

O rendimento quântico máximo do PSII (Fv/Fm) expressa a eficiência na captura da energia de excitação pelos centros de reação abertos do PSII (Krause \& Weiss, 1991), podendo, representar a eficiência quântica do transporte de elétrons através desse fotossistema, sendo também um indicador válido para danos fotoinibitórios.

Em relação à eficiência quântica máxima do Fotossistema II (razão Fv/Fm), poucas diferenças significativas foram encontradas para as duas cultivares estudadas nos anos 2006/2007 e 2007/2008 (Tabela 2). Em todas as épocas avaliadas, a soja M-SOY 7908 RR não apresentou diferença significativa entre os tratamentos, exceto aos 25 DAA e no primeiro ano. Nessas condições, em que a testemunha mantida infestada e a mistura

Tabela 1 - Tratamentos e doses de herbicidas utilizadas nos anos agrícolas 2006/2007 e 2007/2008.

\begin{tabular}{lc}
\multicolumn{1}{c}{ Nome comum } & Dose \\
\cline { 2 - 2 } & $\begin{array}{c}\text { i.a }{ }^{(1)} \text { ou e.a }^{(2)} \\
\left(\mathrm{g} \mathrm{ha}^{-1}\right)\end{array}$ \\
\hline Testemunha mantida infestada & - \\
Testemunha capinada & - \\
Lactofen & 168 \\
Glyphosate & 1080 \\
Lactofen + chlorimuron-ethyl & $96+10$ \\
Chlorimuron-ethyl + imazethapyr & $10+70$ \\
Chlorimuron-ethyl + bentazon & $10+600$ \\
Glyphosate + imazethapyr & $900+70$ \\
Lactofen + chlorimuron-ethyl + imazethapyr & $96+10+70$ \\
Lactofen + chlorimuron-ethyl + imazethapyr / haloxyfop-methyl & $96+10+70 / 60$ \\
\hline
\end{tabular}

${ }^{1}$ Ingrediente ativo. ${ }^{2}$ Equivalente ácido, utilizado para o produto glyphosate. 
chlorimuron-ethyl + imazethapyr apresentaram razão Fv/ Fm com valor superior ao tratamento em que foi aplicado lactofen + chlorimuron-ethyl. Porém, tais valores não são indicativos de estresse, segundo Maxwell \& Johnson (2000), se a planta estiver em condições não estressantes, o valor Fv/FM, na maioria das espécies, pode variar de 0,75 a 0,85 . Os valores inferiores a esses indicarão estresse e redução da eficiência quântica máxima do fotossistema II e, por consequência, do potencial fotossintético da planta. Bjorkman \& Demming (1987), que avaliaram a relação Fv/ Fm em diferentes espécies e ambientes, encontraram um valor médio $\mathrm{Fv} / \mathrm{Fm}$ de 0,83 , resultado similar ao encontrado nesta pesquisa. No segundo ano, não foram observadas diferenças significativas entre os tratamentos.

Tabela 2 - Média da razão $\mathrm{F}_{\mathrm{v}} / \mathrm{F}_{\mathrm{m}}$ em folhas de soja transgênica (M-SOY $7908 \mathrm{RR}$ ), em relação aos dias após a aplicação dos herbicidas, nos anos agrícolas 2006/2007 e 2007/2008.

\begin{tabular}{|c|c|c|c|c|c|}
\hline \multirow[t]{2}{*}{ Tratamento } & \multicolumn{5}{|c|}{$2006 / 2007$} \\
\hline & $4 \mathrm{DAA}$ & $11 \mathrm{DAA}$ & 18 DAA & $25 \mathrm{DAA}$ & $32 \mathrm{DAA}$ \\
\hline $\mathrm{T} 1$ & 0,79 & 0,65 & 0,83 & $0,85 \mathrm{a}$ & 0,83 \\
\hline $\mathrm{T} 2$ & 0,76 & 0,82 & 0,81 & $0,82 a b$ & 0,79 \\
\hline $\mathrm{T} 3$ & 0,69 & 0,82 & 0,83 & $0,82 \mathrm{ab}$ & 0,83 \\
\hline $\mathrm{T} 4$ & 0,79 & 0,82 & 0,82 & $0,82 \mathrm{ab}$ & 0,83 \\
\hline T5 & 0,72 & 0,79 & 0,82 & $0,80 \mathrm{~b}$ & 0,81 \\
\hline T6 & 0,80 & 0,82 & 0,83 & $0,85 \mathrm{a}$ & 0,83 \\
\hline $\mathrm{T} 7$ & 0,78 & 0,78 & 0,82 & $0,82 \mathrm{ab}$ & 0,82 \\
\hline $\mathrm{T} 8$ & 0,77 & 0,83 & 0,84 & $0,84 \mathrm{ab}$ & 0,82 \\
\hline T9 & 0,81 & 0,83 & 0,83 & $0,82 \mathrm{ab}$ & 0,82 \\
\hline $\mathrm{T} 10$ & 0,76 & 0,80 & 0,82 & $0,84 \mathrm{ab}$ & 0,84 \\
\hline $\mathrm{F}_{\text {Trat }}$ & $1,25^{\mathrm{ns}}$ & $1,07^{\mathrm{ns}}$ & $0,54^{\mathrm{ns}}$ & $3,45 * *$ & $1,31^{\mathrm{ns}}$ \\
\hline $\mathrm{CV}(\%)$ & 9,06 & 12,80 & 2,62 & 1,98 & 2,62 \\
\hline DMS (Tukey) & 0,17 & 0,25 & 0,05 & 0,04 & 0,05 \\
\hline \multirow[t]{2}{*}{ Tratamento } & \multicolumn{5}{|c|}{$2007 / 2008$} \\
\hline & $6 \mathrm{DAA}$ & $14 \mathrm{DAA}$ & & $\mathrm{AA}$ & $28 \mathrm{DAA}$ \\
\hline $\mathrm{T} 1$ & 0,74 & 0,82 & & & 0,82 \\
\hline $\mathrm{T} 2$ & 0,78 & 0,81 & & & 0,84 \\
\hline $\mathrm{T} 3$ & 0,79 & 0,81 & & & 0,83 \\
\hline $\mathrm{T} 4$ & 0,77 & 0,81 & & & 0,84 \\
\hline $\mathrm{T} 5$ & 0,79 & 0,73 & & & 0,83 \\
\hline T6 & 0,72 & 0,71 & & & 0,81 \\
\hline $\mathrm{T} 7$ & 0,77 & 0,81 & & & 0,82 \\
\hline $\mathrm{T} 8$ & 0,72 & 0,77 & & & 0,85 \\
\hline $\mathrm{T} 9$ & 0,78 & 0,78 & & & 0,85 \\
\hline $\mathrm{T} 10$ & 0,77 & 0,80 & & & 0,85 \\
\hline $\mathrm{F}_{\text {Trat }}$ & $2,51 *$ & $2,22^{\mathrm{ns}}$ & & & $1,83^{\mathrm{ns}}$ \\
\hline $\mathrm{CV}(\%)$ & 4,35 & 6,37 & & & 2,24 \\
\hline DMS (Tukey) & 0,03 & 0,12 & & & 0,04 \\
\hline
\end{tabular}

$\mathrm{T} 1=$ testemunha mantida infestada. $\mathrm{T} 2=$ testemunha capinada. $\mathrm{T} 3=$ lactofen; $\mathrm{T} 4=$ glyphosate. $\mathrm{T} 5=$ lactofen + chlorimuronethyl. T6 = chlorimuron-ethyl + imazethapyr. $\mathrm{T} 7=$ chlorimuron-ethyl + bentazon. $\mathrm{T} 8=$ glyphosate + imazethapyr. $\mathrm{T} 9=$ lactofen + chlorimuron-ethyl + imazethapyr. T10 = lactofen + chlorimuron-ethyl + imazethapyr/ haloxyfop-methyl

Médias seguidas da mesma letra na coluna não diferem significativamente entre si pelo teste de Tukey, a 5\% de probabilidade. * Significativo ao nível de $5 \%$ de probabilidade. ${ }^{\text {ns }}$ - não significativo. 
Aos 4 DAA e no primeiro ano (Tabela 3), para MSOY 8001, a mistura lactofen + chlorimuron-ethyl + imazethapyr / haloxyfop-methyl proporcionou valor Fv/ Fm inferior ao da testemunha mantida infestada. Enquanto aos 6 DAA, e no segundo ano, a testemunha capinada apresentou valor superior aos das misturas lactofen + chlorimuron-ethyl e lactofen + chlorimuron-ethyl + imazethapyr / haloxyfop-methyl. Tal resposta demonstra sintomas de estresse, o que pode ser confirmado pela aparência das folhas e brotos, que se apresentavam enrugados, com sinais de clorose e necrose resultantes da perda de clorofila. Contudo, com o desenvolvimento da cultura, esses sintomas foram se tornando menos intensos e desaparecendo completamente, sugerindo que o herbicida

Tabela 3 - Média da razão $\mathrm{F}_{\mathrm{v}} / \mathrm{F}_{\mathrm{m}}$ em folhas de soja convencional (M-SOY 8001), em relação aos dias após a aplicação dos herbicidas, nos anos agrícolas 2006/2007 e 2007/2008.

\begin{tabular}{|c|c|c|c|c|c|}
\hline \multirow[t]{2}{*}{ Tratamento } & \multicolumn{5}{|c|}{$2006 / 2007$} \\
\hline & $4 \mathrm{DAA}$ & $11 \mathrm{DAA}$ & $18 \mathrm{DAA}$ & $25 \mathrm{DAA}$ & 32 DAA \\
\hline $\mathrm{T} 1$ & $0,73 \mathrm{a}$ & $0,78 \mathrm{a}$ & $0,84 \mathrm{a}$ & $0,83 \mathrm{a}$ & $0,85 \mathrm{a}$ \\
\hline $\mathrm{T} 2$ & $0,66 \mathrm{ab}$ & $0,81 \mathrm{a}$ & $0,82 \mathrm{a}$ & $0,84 \mathrm{a}$ & $0,84 \mathrm{a}$ \\
\hline $\mathrm{T} 3$ & $0,72 \mathrm{ab}$ & $0,78 \mathrm{a}$ & $0,84 \mathrm{a}$ & $0,84 \mathrm{a}$ & $0,83 \mathrm{a}$ \\
\hline $\mathrm{T} 4$ & $0,00 \mathrm{c}$ & $0,00 \mathrm{~b}$ & $0,00 \mathrm{~b}$ & $0,00 \mathrm{~b}$ & $0 \mathrm{~b}$ \\
\hline $\mathrm{T} 5$ & $0,71 \mathrm{ab}$ & $0,78 \mathrm{a}$ & $0,85 \mathrm{a}$ & $0,85 \mathrm{a}$ & $0,84 \mathrm{a}$ \\
\hline T6 & $0,71 \mathrm{ab}$ & $0,80 \mathrm{a}$ & $0,83 \mathrm{a}$ & $0,83 \mathrm{a}$ & $0,83 \mathrm{a}$ \\
\hline $\mathrm{T} 7$ & $0,69 a b$ & $0,76 \mathrm{~b}$ & $0,84 \mathrm{a}$ & $0,84 \mathrm{a}$ & $0,84 \mathrm{a}$ \\
\hline $\mathrm{T} 8$ & $0,00 \mathrm{c}$ & $0,00 \mathrm{~b}$ & $0,00 \mathrm{~b}$ & $0,00 \mathrm{~b}$ & $0 \mathrm{~b}$ \\
\hline T9 & $0,69 a b$ & $0,77 \mathrm{a}$ & $0,83 \mathrm{a}$ & $0,83 \mathrm{a}$ & $0,83 \mathrm{a}$ \\
\hline $\mathrm{T} 10$ & $0,63 \mathrm{~b}$ & $0,76 \mathrm{a}$ & $0,84 \mathrm{a}$ & $0,84 \mathrm{a}$ & $0,85 \mathrm{a}$ \\
\hline $\mathrm{F}_{\text {Trat }}$ & $223,16 * *$ & $302,10 * *$ & $2071,28 * *$ & $2896,49 * *$ & $2610,17 * *$ \\
\hline $\mathrm{CV}(\%)$ & 7,10 & 6,10 & 2,32 & 1,96 & 2,06 \\
\hline DMS (Tukey) & 0,09 & 0,09 & 0,04 & 0,03 & 0,03 \\
\hline \multirow[t]{2}{*}{ Tratamento } & \multicolumn{5}{|c|}{$2007 / 2008$} \\
\hline & $6 \mathrm{DAA}$ & $14 \mathrm{DAA}$ & & $21 \mathrm{DAA}$ & 28 DAA \\
\hline $\mathrm{T} 1$ & $0,76 \mathrm{ab}$ & $0,70 \mathrm{a}$ & & $0,83 \mathrm{a}$ & $0,83 \mathrm{a}$ \\
\hline $\mathrm{T} 2$ & $0,80 \mathrm{a}$ & $0,76 \mathrm{a}$ & & $0,80 \mathrm{a}$ & $0,86 \mathrm{a}$ \\
\hline $\mathrm{T} 3$ & $0,72 \mathrm{ab}$ & $0,78 \mathrm{a}$ & & $0,81 \mathrm{a}$ & $0,84 \mathrm{a}$ \\
\hline $\mathrm{T} 4$ & $0,00 \mathrm{c}$ & $0,00 \mathrm{~b}$ & & $0,00 \mathrm{~b}$ & $0,00 \mathrm{~b}$ \\
\hline $\mathrm{T} 5$ & $0,66 \mathrm{~b}$ & $0,78 \mathrm{a}$ & & $0,81 \mathrm{a}$ & $0,83 \mathrm{a}$ \\
\hline T6 & $0,77 \mathrm{ab}$ & $0,69 \mathrm{a}$ & & $0,78 \mathrm{a}$ & $0,83 \mathrm{a}$ \\
\hline $\mathrm{T} 7$ & $0,73 \mathrm{ab}$ & $0,77 \mathrm{a}$ & & $0,80 \mathrm{a}$ & $0,85 \mathrm{a}$ \\
\hline $\mathrm{T} 8$ & $0,00 \mathrm{c}$ & $0,00 \mathrm{~b}$ & & $0,00 \mathrm{~b}$ & $0,00 \mathrm{~b}$ \\
\hline T9 & $0,69 \mathrm{ab}$ & $0,69 \mathrm{a}$ & & $0,78 \mathrm{a}$ & $0,84 \mathrm{a}$ \\
\hline $\mathrm{T} 10$ & $0,66 \mathrm{~b}$ & $0,69 \mathrm{a}$ & & $0,78 \mathrm{a}$ & $0,85 \mathrm{a}$ \\
\hline $\mathrm{F}_{\text {Trat }}$ & $114,26 * *$ & $67,63 * *$ & & $287,83 * *$ & $2567,66^{* *}$ \\
\hline $\mathrm{CV}(\%)$ & 9,97 & 12,92 & & 6,22 & 2,08 \\
\hline DMS (Tukey) & 0,14 & 0,18 & & 0,09 & 0,03 \\
\hline
\end{tabular}

$\mathrm{T} 1=$ testemunha sem capina. $\mathrm{T} 2=$ testemunha capinada. $\mathrm{T} 3=$ lactofen. $\mathrm{T} 4=$ glyphosate. $\mathrm{T} 5=$ lactofen + chlorimuron-ethyl. $\mathrm{T} 6=$ chlorimuron-ethyl + imazethapyr. $\mathrm{T} 7=$ chlorimuron-ethyl + bentazon. $\mathrm{T} 8=$ glyphosate + imazethapyr. $\mathrm{T} 9=$ lactofen + chlorimuronethyl + imazethapyr. T10 = lactofen + chlorimuron-ethyl + imazethapyr/ haloxyfop-methyl.

Médias seguidas da mesma letra na coluna não diferem significativamente entre si pelo teste de Tukey, a 5\% de probabilidade.

** - significativo ao nível de $1 \%$ de probabilidade. 
tenha causado dano reversível ao aparelho fotossintético da soja, e que a planta tenha conseguido se recuperar sem sintomas aparentes. As plantas tratadas com glyphosate e glyphosate + imazethapyr morreram.

O aparecimento de sintomas de estresse nos primeiros dias após a aplicação dos herbicidas decorre, provavelmente, da aplicação do lactofen, o qual pertence à classe dos difeniléteres, que inibem a enzima protoporfirinogênio oxidase (PROTOX). Essa enzima converte o protoporfirinogênio-IX em protoporfirina-IX, a qual produz clorofila e citocromos a partir de reações com $\mathrm{Mg}$ e Fe, respectivamente (Matringe et al., 1989). A ação do herbicida acontece pela competição com a enzima PROTOX, impedindo a produção de protoporfirina-IX e resultando em acúmulo de protoporfirinogênio no cloroplasto. A partir desse acúmulo, protoporfirinogênio-IX se difunde para o citoplasma onde é rapidamente convertido para protoporfirina IX, por uma enzima peroxidase que é insensível ao herbicida (Jacobs \& Jacobs, 1993). A protoporfirina-IX é um composto fotodinâmico e seu acúmulo no citoplasma, quando em presença de luz e oxigênio molecular, origina oxigênio singleto (Merotto Junior \& Vidal, 2001). Ainda, nos primeiros dias após a aplicação do herbicida, esse radical livre, que é altamente reativo, provoca a peroxidação dos lipídios das membranas (Becerril \& Duke, 1989), desestruturando-as e acelerando o processo de aparecimento dos sintomas típicos como clorose e necrose dos tecidos, pela redução do teor de clorofila.

$\mathrm{Na}$ Tabela 4, nos dois anos, podem-se observar os valores médios correspondentes às leituras do medidor portátil de clorofilas (MPC) em folhas de soja M-SOY 7908 RR. Entre os tratamentos e aos 4 e 11 DAA, no primeiro ano, e aos 6 e 14 DAA, no segundo, foram observadas diferenças significativas. Aos 4 DAA, o lactofen aplicado isolado, quando comparado à testemunha mantida infestada, proporcionou redução de $12 \%$ nos valores do MPC. Aos 11 DAA, as misturas lactofen + chlorimuronethyl e lactofen + chlorimuron-ethyl + imazethapyr + haloxifop-methyl resultaram em valores de MPC de $11 \%$ inferior ao encontrado na testemunha capinada. Esses resultados sugerem que o lactofen ocasionou inibição de Protox, uma enzima envolvida na biossíntese de citocromos e clorofila (Dan Hess, 2000), resultando em sintomas de estresse (aparecimento de bronzeamento e clorose nas folhas). Aos 6 DAA, quando comparada à testemunha capinada, a mistura chlorimuron-ethyl + imazethapyr proporcionou redução no valor do MPC. Segundo Trezzi \& Vidal (2001), o chlorimuron-ethyl (do grupo químico das sulfoniluréias) e o imazethapyr (do grupo químico das imidazolinonas) são inibidores da enzima acetolactato sintase (ALS), e essa enzima é responsável por catalisar a síntese de três aminoácidos essenciais: valina, leucina e isoleucina. Essa inibição interrompe a síntese protéica que, por sua vez, interfere na síntese do DNA e no crescimento celular. Esse comprometimento no crescimento celular ficou constatado por meio da interrupção temporária do crescimento das plantas de soja (dados não mostrados), assim como foi verificado murcha e clorose das folhas dessas plantas.

Aos 14 DAA, houve diferença significativa entre as testemunhas (capinada e mantida infestada) e glyphosate aplicado isolado, e os tratamentos lactofen + chlorimuronethyl + imazethapyr e lactofen + chlorimuron-ethyl + imazethapyr/haloxyfop-methyl. Os dois últimos tratamentos resultaram em um valor do MPC inferior aos três primeiros tratamentos, ocasionando sintomas de estresse visuais nas plantas, tais como: interrupção temporária do crescimento, folhas murchas e cloróticas (dados não mostrados), os quais desapareceram com a idade das plantas. Já aos 18; 25 e 32 DAA no primeiro ano, e aos 21, 28 e 35 DAA no segundo ano, não foi observada diferença significativa entre os herbicidas aplicados e os tratamentos testemunhas. Nos dois anos de estudo, para o cultivar transgênico, e nas duas primeiras épocas de avaliação, foram observados os sintomas de estresse induzidos pela aplicação dos herbicidas. Entretanto, com a idade das plantas, esses sintomas desapareceram completamente.

No primeiro ano, para a soja convencional (Tabela 5), desconsiderando-se os tratamentos em que foram aplicados glyphosate isolado e em mistura com imazethapyr, os quais ocasionaram a morte das plantas, apenas aos 18 e 25 DAA, foi observada diferença significativa nos valores do MPC. Aos 18 DAA e nas folhas dos tratamentos testemunha mantida infestada, testemunha capinada e a mistura lactofen + chlorimuron-ethyl, as leituras do MPC foram superiores quando comparados aos tratamentos em que houve aplicação de lactofen e lactofen + chlorimuron-ethyl + imazethapyr/haloxifop-methyl. Nessa condição, quando comparado à testemunha capinada, houve redução no valor da leitura do MPC em torno de $13 \%$. Porém, os três primeiros tratamentos citados, não apresentaram diferença estatística quando comparados com os tratamentos chlorimuron-ethyl + imazethapyr, chlorimuron-ethyl + bentazon e lactofen + chlorimuron-ethyl + imazethapyr. A leitura do MPC, aos 25 DAA, para o tratamento testemunha mantida infestada e a mistura lactofen + chlorimuron-ethyl, diferiu do tratamento em que foi aplicado lactofen + chlorimuron-ethyl + imazethapyr/haloxifop-methyl. Nessa condição, também houve redução de $13 \%$, quando comparado à testemunha mantida infestada. 
No segundo ano, a resposta da cultivar foi de maneira diferenciada, pois desde a primeira avaliação, os tratamentos apresentaram diferenças entre si. Aos 6 DAA, a testemunha capinada apresentou diferença significativa dos tratamentos lactofen aplicado isolado e das misturas chlorimuron-ethyl + imazethapyr, lactofen + chlorimuronethyl + imazethapyr e lactofen + chlorimuron-ethyl + imazethapyr/ haloxifop-methyl com redução na leitura do
MPC de 28; 20; 22 e 29\%, respectivamente. Nesse caso, houve visíveis sintomas de clorose dos tecidos (dados não mostrados). Aos 14 DAA, as testemunhas (capinada e mantida infestada) diferiram significativamente do tratamento em que se aplicou o lactofen aplicado isolado, e das misturas lactofen + chlorimuron-ethyl + imazethapyr, e lactofen + chlorimuron-ethyl + imazethapyr/haloxifopmethyl. Aos 21 DAA, as testemunhas (capinada e mantida

Tabela 4 - Média dos valores das leituras do medidor portátil de clorofilas em folhas de soja transgênica (M-SOY 7908 RR), em relação aos dias após a aplicação dos herbicidas, nos anos agrícolas 2006/2007 e 2007/2008.

\begin{tabular}{|c|c|c|c|c|c|}
\hline \multirow{2}{*}{ Tratamento } & \multicolumn{5}{|c|}{$2006 / 2007$} \\
\hline & 4 DAA & 11 DAA & 18 DAA & 25 DAA & 32 DAA \\
\hline $\mathrm{T} 1$ & $40,40 \mathrm{a}$ & $42,4 \mathrm{ab}$ & 43,10 & 42,70 & 44,30 \\
\hline $\mathrm{T} 2$ & $39,20 \mathrm{ab}$ & $44,07 \mathrm{a}$ & 43,35 & 41,60 & 44,90 \\
\hline $\mathrm{T} 3$ & $35,62 \mathrm{~b}$ & $41,25 \mathrm{ab}$ & 41,52 & 40,87 & 43,72 \\
\hline $\mathrm{T} 4$ & $39,82 \mathrm{ab}$ & $41,17 \mathrm{ab}$ & 42,05 & 42,27 & 43,47 \\
\hline T5 & $37,62 \mathrm{ab}$ & $39,12 \mathrm{~b}$ & 39,85 & 40,00 & 42,55 \\
\hline T6 & $37,82 \mathrm{ab}$ & $41,27 \mathrm{ab}$ & 42,27 & 43,82 & 45,20 \\
\hline $\mathrm{T} 7$ & $39,42 \mathrm{ab}$ & $42,37 \mathrm{ab}$ & 43,37 & 42,72 & 45,82 \\
\hline $\mathrm{T} 8$ & $39,82 \mathrm{ab}$ & $41,25 \mathrm{ab}$ & 43,25 & 42,02 & 44,07 \\
\hline T9 & $39,02 \mathrm{ab}$ & $41,15 \mathrm{ab}$ & 42,50 & 42,95 & 44,35 \\
\hline $\mathrm{T} 10$ & $38,72 \mathrm{ab}$ & $39,17 \mathrm{~b}$ & 39,20 & 40,75 & 41,35 \\
\hline $\mathrm{F}_{\text {Trat }}$ & $2,04^{\mathrm{ns}}$ & $3,77 * *$ & $2,75 *$ & $1,75^{\mathrm{ns}}$ & $1,44^{\mathrm{ns}}$ \\
\hline $\mathrm{CV}(\%)$ & 5,06 & 3,65 & 4,22 & 4,22 & 4,93 \\
\hline DMS (Tukey) & 4,76 & 3,67 & 4,32 & 4,30 & 5,27 \\
\hline \multirow{2}{*}{ Tratamento } & \multicolumn{5}{|c|}{$2007 / 2008$} \\
\hline & 6 DAA & $14 \mathrm{DAA}$ & $21 \mathrm{DAA}$ & $28 \mathrm{DAA}$ & $35 \mathrm{DAA}$ \\
\hline $\mathrm{T} 1$ & $30,90 \mathrm{ab}$ & $41,92 \mathrm{a}$ & 38,77 & 44,65 & 44,50 \\
\hline $\mathrm{T} 2$ & $34,37 \mathrm{a}$ & $43,02 \mathrm{a}$ & 41,05 & 45,87 & 45,60 \\
\hline $\mathrm{T} 3$ & $30,25 \mathrm{ab}$ & $34,32 \mathrm{ab}$ & 32,90 & 43,82 & 43,72 \\
\hline $\mathrm{T} 4$ & $31,40 \mathrm{ab}$ & $43,22 \mathrm{a}$ & 40,82 & 46,40 & 46,25 \\
\hline $\mathrm{T} 5$ & $29,46 \mathrm{ab}$ & $38,65 \mathrm{ab}$ & 37,42 & 44,02 & 43,97 \\
\hline T6 & $24,90 \mathrm{~b}$ & $35,07 \mathrm{ab}$ & 34,40 & 43,27 & 43,45 \\
\hline $\mathrm{T} 7$ & $32,72 \mathrm{ab}$ & $39,67 \mathrm{ab}$ & 39,80 & 45,40 & 45,37 \\
\hline $\mathrm{T} 8$ & $31,60 \mathrm{ab}$ & $39,27 \mathrm{ab}$ & 35,97 & 44,82 & 44,80 \\
\hline $\mathrm{T} 9$ & $30,10 \mathrm{ab}$ & $32,30 \mathrm{~b}$ & 36,80 & 43,65 & 43,10 \\
\hline $\mathrm{T} 10$ & $31,80 \mathrm{ab}$ & $32,25 \mathrm{~b}$ & 34,12 & 44,00 & 43,77 \\
\hline $\mathrm{F}_{\text {Trat }}$ & $2,71 *$ & $5,19 * *$ & $2,09^{\mathrm{ns}}$ & $1,80^{\mathrm{ns}}$ & $2,06^{\mathrm{ns}}$ \\
\hline $\mathrm{CV}(\%)$ & 9,61 & 9,76 & 10,71 & 3,43 & 3,23 \\
\hline DMS (Tukey) & 7,16 & 9,01 & 9,69 & 3,72 & 3,49 \\
\hline
\end{tabular}

$\mathrm{T} 1=$ testemunha mantida infestada. $\mathrm{T} 2=$ testemunha capinada. $\mathrm{T} 3=$ lactofen. $\mathrm{T} 4=$ glyphosate. $\mathrm{T} 5=$ lactofen + chlorimuronethyl. T6 = chlorimuron-ethyl + imazethapyr. T7 $=$ chlorimuron-ethyl + bentazon. T8 = glyphosate + imazethapyr. T9 $=$ lactofen + chlorimuron-ethyl + imazethapyr. T10 = lactofen + chlorimuron-ethyl + imazethapyr/ haloxyfop-methyl.

Médias seguidas da mesma letra na coluna não diferem significativamente entre si pelo teste de Tukey, a $5 \%$ de probabilidade.

* - significativo ao nível de $5 \%$ de probabilidade. ${ }^{* *}$ - significativo ao nível de $1 \%$ de probabilidade. ${ }^{\text {ns }}$ - não significativo. 
infestada) diferiram significativamente de lactofen aplicado isolado, e das misturas chlorimuron-ethyl + imazethapyr; lactofen + chlorimuron-ethyl + imazethapyr e lactofen + chlorimuron-ethyl + imazethapyr/haloxifop- methyl. Aos 28 DAA, as testemunhas apresentaram diferença significativa apenas da mistura chlorimuronethyl + imazethapyr com redução na leitura do MPC de 25 e $31 \%$, respectivamente.

Tabela 5 - Média dos valores das leituras do medidor portátil de clorofilas em folhas de soja convencional (M-SOY 8001), em relação aos dias após a aplicação dos herbicidas, nos anos agrícolas 2006/2007 e 2007/2008.

\begin{tabular}{|c|c|c|c|c|c|}
\hline \multirow{2}{*}{ Tratamento } & \multicolumn{5}{|c|}{$2006 / 2007$} \\
\hline & 4 DAA & $11 \mathrm{DAA}$ & 18 DAA & 25 DAA & $32 \mathrm{DAA}$ \\
\hline T1 & $37,90 \mathrm{a}$ & $42,00 \mathrm{a}$ & $41,52 \mathrm{a}$ & $39,97 \mathrm{a}$ & $44,10 \mathrm{a}$ \\
\hline $\mathrm{T} 2$ & $40,02 \mathrm{a}$ & $42,50 \mathrm{a}$ & $41,62 \mathrm{a}$ & $39,00 \mathrm{ab}$ & $42,12 \mathrm{a}$ \\
\hline $\mathrm{T} 3$ & $36,07 \mathrm{a}$ & 36,85 a & $36,27 \mathrm{~b}$ & $36,20 a b$ & $41,87 \mathrm{a}$ \\
\hline $\mathrm{T} 4$ & $0,00 \mathrm{~b}$ & $0,00 \mathrm{~b}$ & $0,00 \mathrm{c}$ & $0,00 \mathrm{c}$ & $0,00 \mathrm{~b}$ \\
\hline T5 & $34,75 \mathrm{a}$ & 36,95 a & $40,55 \mathrm{a}$ & 39,65 a & $42,25 \mathrm{a}$ \\
\hline T6 & $36,50 \mathrm{a}$ & $40,80 \mathrm{a}$ & $40,25 \mathrm{ab}$ & $36,97 \mathrm{ab}$ & $41,92 \mathrm{a}$ \\
\hline $\mathrm{T} 7$ & 35,87 a & $40,07 \mathrm{a}$ & $37,67 \mathrm{ab}$ & $37,62 \mathrm{ab}$ & $43,75 \mathrm{a}$ \\
\hline $\mathrm{T} 8$ & $0,00 \mathrm{~b}$ & $0,00 \mathrm{~b}$ & $0,00 \mathrm{c}$ & $0,00 \mathrm{c}$ & $0,00 \mathrm{~b}$ \\
\hline T9 & $36,25 \mathrm{a}$ & $37,10 \mathrm{a}$ & $38,00 \mathrm{ab}$ & $36,97 a b$ & $41,80 \mathrm{a}$ \\
\hline $\mathrm{T} 10$ & $36,87 \mathrm{a}$ & $39,15 \mathrm{a}$ & $36,22 \mathrm{~b}$ & $34,90 \mathrm{~b}$ & $42,15 \mathrm{a}$ \\
\hline $\mathrm{F}_{\text {Trat }}$ & $176,92 * *$ & $171,78 * *$ & $395,29 * *$ & $315,04 * *$ & $302,18 * *$ \\
\hline $\mathrm{CV}(\%)$ & 7,96 & 8,10 & 5,34 & 5,97 & 6,07 \\
\hline DMS (Tukey) & 5,69 & 6,21 & 4,05 & 4,37 & 5,01 \\
\hline \multirow{2}{*}{ Tratamento } & \multicolumn{5}{|c|}{$2007 / 2008$} \\
\hline & 6 DAA & 14 DAA & $21 \mathrm{DAA}$ & $28 \mathrm{DAA}$ & $35 \mathrm{DAA}$ \\
\hline $\mathrm{T} 1$ & $31,85 \mathrm{abcd}$ & $40,37 \mathrm{a}$ & $40,62 \mathrm{a}$ & $39,05 \mathrm{a}$ & $41,05 \mathrm{a}$ \\
\hline $\mathrm{T} 2$ & $35,92 \mathrm{a}$ & $39,82 \mathrm{a}$ & $42,27 \mathrm{a}$ & $42,27 \mathrm{a}$ & 39,42 a \\
\hline $\mathrm{T} 3$ & $26,00 \mathrm{~cd}$ & $27,55 \mathrm{~d}$ & $31,20 \mathrm{bc}$ & $36,57 \mathrm{ab}$ & $40,07 \mathrm{a}$ \\
\hline $\mathrm{T} 4$ & $0,00 \mathrm{e}$ & $0,00 \mathrm{e}$ & $0,00 \mathrm{~d}$ & $0,00 \mathrm{c}$ & $0,00 \mathrm{~b}$ \\
\hline T5 & $33,67 \mathrm{ab}$ & $35,12 a b c$ & $36,72 \mathrm{abc}$ & $35,97 \mathrm{ab}$ & 39,47 a \\
\hline T6 & $28,57 \mathrm{bcd}$ & $34,35 \mathrm{abc}$ & $30,27 \mathrm{c}$ & $29,07 \mathrm{~b}$ & $39,12 \mathrm{a}$ \\
\hline $\mathrm{T} 7$ & $32,65 \mathrm{abc}$ & $37,87 \mathrm{ab}$ & $37,55 \mathrm{ab}$ & $37,62 a b$ & 40,62 a \\
\hline $\mathrm{T} 8$ & $0,00 \mathrm{e}$ & $0,00 \mathrm{e}$ & $0,00 \mathrm{~d}$ & $0,00 \mathrm{c}$ & $0,00 \mathrm{~b}$ \\
\hline T9 & $27,95 \mathrm{bcd}$ & $32,17 \mathrm{bcd}$ & $31,70 \mathrm{bc}$ & $35,40 \mathrm{ab}$ & $39,12 \mathrm{a}$ \\
\hline $\mathrm{T} 10$ & $25,35 \mathrm{~d}$ & $29,85 \mathrm{~cd}$ & $32,52 \mathrm{bc}$ & $34,42 \mathrm{ab}$ & $40,45 \mathrm{a}$ \\
\hline $\mathrm{F}_{\text {Trat }}$ & $88,49 * *$ & $137,82 * *$ & $110,11 * *$ & $66,68 * *$ & $472,33 * *$ \\
\hline $\mathrm{CV}(\%)$ & 11,59 & 9,32 & 10,41 & 13,22 & 4,85 \\
\hline DMS (Tukey) & 6,82 & 6,28 & 7,16 & 9,33 & 3,77 \\
\hline
\end{tabular}

$\mathrm{T} 1=$ testemunha sem capina. $\mathrm{T} 2=$ testemunha capinada. $\mathrm{T} 3=$ lactofen. $\mathrm{T} 4=$ glyphosate. $\mathrm{T} 5=$ lactofen + chlorimuron-ethyl. $\mathrm{T} 6=$ chlorimuron-ethyl + imazethapyr. $\mathrm{T} 7=$ chlorimuron-ethyl + bentazon. $\mathrm{T} 8=$ glyphosate + imazethapyr. $\mathrm{T} 9=$ lactofen + chlorimuronethyl + imazethapyr. T10 = lactofen + chlorimuron-ethyl + imazethapyr/ haloxyfop-methyl.

Médias seguidas da mesma letra na coluna não diferem significativamente entre si pelo teste de Tukey, a 5\% de probabilidade. ** - significativo ao nível de $1 \%$ de probabilidade. 


\section{CONCLUSÕES}

As misturas lactofen + chlorimuron-ethyl e lactofen + chlorimuron-ethyl + imazethapyr / haloxifop-methyl ocasionaram redução na eficiência fotossintética máxima da cultivar convencional.

As duas cultivares (convencional e transgênica) apresentaram suscetibilidade ao lactofen aplicado isolado e em mistura com chlorimuron-ethyl, imazethapyr e haloxifop-methyl, com redução nos teores de clorofila, estimados pelo Medidor Portátil de Clorofilas.

\section{AGRADECIMENTOS}

À Universidade Estadual do Maranhão, pela concessão de bolsa de doutorado.

\section{REFERÊNCIAS BIBLIOGRÁFICAS}

ARGENTA, G.; SILVA, P.R.F. da; BORTOLINI, C.G.; FORSTHOFER, E.L.; STRIEDER, M.L. Relação da leitura do clorofilômetro com os teores de clorofila extraível e de nitrogênio na folha de milho. Revista Brasileira Fisiologia Vegetal, Londrina, v.13, n.2, p.158-116, 2001.

BECERRIL, J.M.; DUKE, S.O. Protoporphyrin IX content correlates with activity of photobleanching herbicides. Plant Physiology, Washington, v.90, n.4, p.1175-1181, 1989.

BENETT, C. G. S.; BUZETTI, S.; SILVA, K. S.; BERGAMASCHINE, A. F.; FABRICIO, J. A.

Produtividade e composição bromatológica do capimmarandu a fontes e doses de nitrogênio. Ciência Agrotecnologia, Lavras, v. 32, n. 5, p. 1629-1636, set./ out., 2008.

BJORKMAN, O.; DEMMIG, B. Photon yield of $\mathrm{O}_{2}$ evolution and chlorophyll fluorescence characteristics at $77 \mathrm{~K}$ among vascular plants of diverse origins. Planta, Berlin, v.170, n.4, p.489-504, 1987.

CATUNDA, M.G.; FREITAS, S.P.; OLIVEIRA, J.G.; SILVA, C.M.M. Efeitos de herbicidas na atividade fotossintética e no crescimento de abacaxi (Ananas comosus). Planta Daninha, Viçosa, v.23, n.1, p.115-121, 2005.

DAN HESS, F. Light-dependent herbicides: an overwiew. Weed Science, Lawrence, v.48, n.2, p.160-170, 2000.

GLYNN, P.; FRASER, C.; GILLIAN, A. Foliar salt tolerance of Acer genotypes using chlorophyll fluorescence. Journal Arboriculture, v.29, p.61-65, 2003.
HERBICIDE RESISTANCEACTION COMMITTEE. Classification of herbicides according to mode of action. Disponível em: <http://www.haracglobal.comh Publication/ClassificationofHerbicideModeof Actiond tabid/222/Default.aspx $>$. Acesso em: 30 maio 2008 .

JACOBS, J.M.; JACOBS, N.M. Porphyrin accumulation and export by isolated barley (Hordeum vulgare) plastids. Plant Physiology, San Diego, v.101, n.4, p.1181-1187, 1993.

KRAUSE, G.H.; WEISS, E. Chlorophyll fluorescence and photosynthesis: the basics. Annual Review Plant Physiology Plant Molecular Biology, Palo Alto, v.42, p.313-349, 1991.

MATRINGE, M.J.; CAMADRO, J.; LABETTE, P.; SCALLA, R. Protoporphyrogen oxidase as a molecular target for diphenyl ether herbicides. Biochemistry Journal, v.260, p.231-235, 1989.

MAXWEEL, K.; JOHNSON, G. Chlorophyll fluorescence: a practical guide. Journal Experimental Botany, Almsford, v.51, p.659-668, 2000.

MELHORANÇA, A.L.; PEREIRA, F.A.R. Eficiência do herbicida lactofen no controle de Euphorbia heterophylla resistente aos herbicidas inibidores da enzima acetolactato sintase (ALS). Revista Brasileira Herbicidas, Brasília, v.1, n.1, p.53-56, 2000.

MEROTTO JÚNIOR, A.; VIDAL, R.A. Herbicidas inibidores da Protox. In: Herbicidologia. Porto Alegre: Artmed, 2001. p.69-86.

OGREN, E.; OQUIST, G. Effect of drought on photosynthesis, chlorophyll florescence and photoinhibition susceptibility in intact willow leaves. Planta, Berlin, v.166, n.3, p.380-388, 1985.

RITCHIE, S.W.; HANWAY, J.J.; THOMPSON, H.E.; BENSON, G.O. How a soybean plant develops. Ames: Yowa State University of Science and Technology, 1977. 20p. (Special Report, 53).

ROZANE, D. E.; SOUZA, H. A. de.; PRADO, R. de M.; NATALE, W.; FRANCO, C. F.; LEAL, R. M. Influência do cultivar, do tipo de folha e do tempo de cultivo na medida indireta da clorofila (SPAD) em mudas de goiabeira. Ciência Agrotecnologia, Lavras, v. 33, n. 6, p. 1538-1543, Nov./dez., 2009. 
SILVEIRA, P.M.; BRAZ, A.J.B.P.; DIDONET, A.D. Uso do clorofilômetro como indicador da necessidade de adubação nitrogenada no feijoeiro. Pesquisa Agropecuária Brasileira, Brasília, v.38, n.9, p.1083-1087, 2003.

TREZZI, M.M.; VIDAL, R.A. Herbicidas inibidores de ALS. In: VIDAL, R.A.; MEROTTO JÚNIOR, A. (Ed.). Herbicidologia. Porto Alegre: Artmed, 2001. p.25-36.

ZANANDREA, I.; NASSI, F. de L.; TURCHETTO, A.C.; BRAGA, E.J.B.; PETERS, J.A.; BACARIN,
M.A. Efeito da salinidade sob parâmetros de fluorescência em Phaseolus vulgaris. Revista Brasileira Agrociência, Pelotas, v.12, n.2, p.157-161, 2006.

ZOTARELLI, L.; CARDOSO, E.G.; PICCININ, J.L.; URQUIAGA, S.; BODDEY, R.M.; TORRES, E.; ALVES, B.J.R. Calibração do medidor de clorofila SPAD-502 para avaliação do conteúdo de nitrogênio do milho. Pesquisa Agropecuária Brasileira, Brasília, v.38, n.9, p.1117-1192, 2003. 